\section{Making a hard cell}

\section{By Lev Osherovich, Senior Writer}

A trio of papers suggests that transforming one differentiated cell type into another can be accomplished without the need for pluripotent, stem cell-like intermediates. Stem cell companies, however, don't think their technologies will be obviated by these discoveries because the new techniques face delivery, dosage and safety challenges that are more complex than those of typical stem cell therapies.

The papers, all published in Nature, show that mouse muscle precursor cells and pancreatic exocrine cells can be coaxed to transdifferentiate into brown adipose tissue ${ }^{1,2}$ and islet $\beta$-cells, ${ }^{3}$ respectively. Brown fat could be useful in treating obesity and type 2 diabetes because brown fat cells burn fat, generating heat in the process. The insulin-secreting $\beta$-cells could be useful in both type 1 and type 2 diabetes.

"These papers reset the thinking" in the cell-therapy field, said Philip Gregory, VP of research at Sangamo BioSciences Inc. "Until now, people have thought that to reprogram a cell you would have to revert it back to a pluripotent state."

Trans fat

In the first two papers, two independent teams at Harvard Medical School showed how muscle precursor cells called skeletal myoblasts can transdifferentiate into brown adipose tissue (BAT). Although human BAT shrinks after infancy to barely detectable levels in adults, increasing BAT levels is a potential strategy to increase energy expenditure and thus fight obesity.

One study, from a team led by Bruce Spiegelman, professor of cell biology at the Dana-Farber Cancer Institute and Harvard Medical School, used cultured mouse cells to show how a transcription factorPR domain containing 16 (PRDM16) - controls BAT development. ${ }^{4}$ The team had previously identified PRDM16 as a BAT biomarker, but its causal role in BAT development was uncertain. ${ }^{4}$

The team found that cultured myoblasts treated with an adenoviral vector expressing PRDM16 developed into BAT cells, in contrast to the muscle cells that arose when the myoblasts were treated with empty vectors. Conversely, knocking down PRDM16 in cultured brown preadipocytes caused them to differentiate into skeletal muscle cells.

"PRDM16 is quite a powerful regulator of brown fat development," said Patrick Seale, a research fellow at Dana-Farber and lead author of the study. "Expression of this single factor can turn on all of the components of brown fat when you express it in the muscles."

Spiegelman's team went on to show that PRDM16 stimulates peroxisome proliferation-activated receptor- $\gamma$ (PPAR- $\gamma$ ), a transcription factor that promotes adipocyte development and energy utilization. That target, which Spiegelman codiscovered in 1994, is agonized by two marketed diabetes drugs: Avandia rosiglitazone from GlaxoSmithKline plc and Actos pioglitazone from Takeda Pharmaceutical Co. Ltd.

The new findings suggest that PRDM16 and BAT could aid the action of these PPAR- $\gamma$ agonists or could themselves be targets. Agonizing PRDM16 or overexpressing it could promote brown adipose tissue formation, which could be useful in treating obesity or diabetes, said Seale.

However, because adult humans have very little BAT, it is unknown whether boosting BAT could indeed stimulate energy use or would even be safe.

Seale's next step is to test whether the higher levels of BAT lead to more energy expenditure. He also plans to transplant cultured myoblasts with extra PRDM16 into mouse models of obesity to determine whether in vitro-generated BAT cells can survive in vivo and increase fat burning.

Spiegelman and Seale's work is the subject of a pending patent and is available for licensing.
"This is a stunning result. I'm amazed that it worked - Jonathan Slack, University of Minnesota

\section{Closer to the bone}

A second study from a team led by Yu-Hua Tseng, assistant investigator at the Joslin Diabetes Center at Harvard Medical School, described a complementary approach to boosting BAT using a secreted morphogenic factor called bone morphogenic protein 7

(BMP7; OP-1). ${ }^{2}$

BMP7 is marketed by Stryker Corp. under a Humanitarian Device Exemption for nonunion tibial fractures and other bone fractures.

Tseng's team found that cultured mouse preadipocytes treated with BMP7 differentiated into BAT cells, whereas untreated controls or cells treated with other bone morphogenic proteins remained unaltered. When the resulting BAT cells were transplanted into mice, they developed into brown fat deposits.

BMP7 knockout mice developed markedly less BAT than wild-type controls. Moreover, mice injected with BMP7 developed additional BAT deposits and showed higher levels of PRDM16 and mitochondrial activity, which led to them having higher energy expenditure and lower body weight than mock-treated controls.

According to Tseng, BMP7 acts upstream of PRDM16 and other BAT-promoting transcription factors and triggers the differentiation of BAT cells from as yet unidentified progenitor cells.

"There is some evidence that the fully differentiated white adipose cells can in fact be induced to convert to brown adipose tissue," said Tseng. Consequently, she believes BMP7 may see a new application as an obesity therapeutic if indeed the fat-accumulating white adipocytes 
could be transdifferentiated directly into fat-burning brown adipocytes.

"In order for brown fat to burn energy, it needs a source, and white adipose tissue is the depot that stores extra energy," said Tseng. "By creating additional brown fat, we could help to burn this extra energy."

To test this hypothesis, Tseng plans to administer BMP7 to obese mice and measure whether it triggers weight loss.

Mitchell Lazar, director of the Institute of Diabetes, Obesity and Metabolism at the University of Pennsylvania, is skeptical. "We have no reason to believe that having more BAT would be of benefit" in obesity and diabetes, he told SciBX.

Lazar said previous efforts to increase human BAT activity by stimulation of adrenergic receptor $\beta 3$ or mitochondrial uncoupling proteins did not result in weight loss.

One effect of enhancing BAT activity would be an increase in body temperature, according to Lazar. "Would people tolerate sitting around and sweating all of the time?" he asked.

In contrast, Jasbir Seehra, CSO at Acceleron

Pharma Inc., thinks it's worth testing BMP7 therapeutics in metabolic disease.

He told SciBX that the morphogen has already been extensively characterized in clinical trials for bone indications and is both safe and effective in that setting. In addition, the good translational track record for BMP7 and related proteins suggests that the morphogen may work similarly in mouse and man.

"BMP7 is extremely well conserved among the species, as are the activin receptors that it uses," Seehra said.

The biggest challenge for BMP7-based obesity therapeutics would be devising a means of systemic delivery that would not lead to ectopic bone formation, he told SciBX.

Acceleron is developing antagonists of several morphogens related to BMP7 to treat bone loss, cancer and metabolic indications. Acceleron's ACE-011 activin receptor type 2A antagonist is in Phase Ib testing for bone loss.

Tseng's findings are the subject of pending patents and are available for licensing from the Joslin Diabetes Center.

\section{Beta max}

In the third paper, another Harvard group, led by Douglas Melton, professor of natural sciences, showed how a different set of transcription factors drives the transdifferentiation of pancreatic exocrine cells into insulin-producing $\beta$-cells. ${ }^{3}$ The findings illustrate that transcriptional reprogramming can occur in vivo, bringing the technique closer to clinical application.

Melton's team found that concurrent adenovirus expression of three transcription factors in the mouse pancreas-neurogenin 3 (Neurog3), pancreatic and duodenal homeobox $1(\mathrm{Pdx} 1)$ and $\mathrm{v}$-maf musculoaponeurotic fibrosarcoma oncogene homolog A (avian) (Mafa) - triggered the appearance of insulin-secreting cells.

According to cell-labeling experiments, the new insulin-producing cells arose from exocrine cells but did not show signs of having undergone cell division. This suggests that the exocrine cells transdifferentiated instead of reverting to a pluripotent stem cell-like state first.
Despite the enhancement of $\beta$-cell numbers, systemic insulin levels in the mice increased only modestly, perhaps because the new cells were not located in the same part of the pancreas as natural $\beta$-cells and did not organize into islets. However, the mice had markedly lower blood sugar than mock-transfected controls, suggesting that even a little more insulin could have a significant biological effect.

"This is a stunning result," said Jonathan Slack, director of the Stem Cell Institute at the University of Minnesota, who made previous key discoveries in the transdifferentiation field. "I'm amazed that it worked so well."

E. Edward Baetge, VP and CSO at Novocell Inc., thinks Melton's in vivo transdifferentiation of therapeutically useful cells in the appropriate organ is a major technical advance.

Previous work showed that cells in the liver could be reprogrammed into insulin-producing cells with a single transcription factor, which he said was a proof of principle. ${ }^{6}$ Until Melton's study, however, "nobody has been able to show reprogramming in the pancreas and in such a short time frame," said Baetge.

Novocell is developing methods to induce the differentiation of human embryonic stem cells into $\beta$-cells. The company's polyethylene glycol encapsulation technology for $\beta$-cell implants is in a Phase I/II trial for type 1 diabetes.

Baetge told SciBX that choosing the right vector for overexpression of $\beta$-cell-promoting transcription factors and ensuring long-term effectiveness will be major challenges.

Other challenges to developing $\beta$-cell transdifferentiation therapies include the immunological complexity of the pancreas and the difficulty of culturing human $\beta$-cells, according to Baetge. Repeating Melton's findings in porcine or nonhuman primate models of diabetes would be the logical next step, he said.

Finally, even if the transdifferentiation technology works, the clinical outcomes may be underwhelming. Baetge noted that transdifferentiation of exocrine cells into $\beta$-cells could trigger autoimmune destruction of the new cells in type 1 diabetes patients. Thus, for now, the technique may be more applicable to type 2 diabetes, in which autoimmunity is not a problem but natural $\beta$-cells can't produce enough insulin to control hyperglycemia.

Sangamo's Gregory was optimistic that Melton's pancreatic findings would eventually translate into therapeutics. He noted that transient expression of the $\beta$-cell-promoting transcription factors from a viral vector yielded long-lasting transdifferentiation and insulin secretion.

"The effects observed were quite durable for many months after the transcription factors have been done and gone," said Gregory. This durability would allow drug developers to bypass some of the issues with gene therapy, such as achieving stable, long-term gene expression, he added.

Sangamo is developing zinc finger transcription factors as therapeutic agents to increase gene expression. Sangamo's SB-509, a plasmid that encodes a transcription factor that boosts expression of VEGF-A, is in Phase II trials to treat diabetic neuropathy. 


\section{Fat chance}

Stem cell companies polled by SciBX did not think that interest in their approaches was likely to decrease. In addition to the head start that stem cell therapies already have, biotechs in that space think transdifferentiation-based therapeutics could have a higher safety bar.

Silviu Itescu, executive director and founder of Mesoblast Ltd., told SciBX that overexpressing or activating transcription factors to alter cell types to treat metabolic diseases would perhaps face extra regulatory scrutiny because of the poor track record of gene-therapy trials involving viral vectors.

"Once you introduce overexpression, you raise a whole new level of complexity to the regulatory process," he said.

Itescu suggested that introducing adult stem cells in situ could achieve the same goals without the need for transfecting cells with viral vectors. He also noted that adult stem cells produce a range of growth factors and morphogens and could promote transdifferentiation of neighboring cells into BAT or $\beta$-cells, but with a more nuanced effect than forced expression of a handful of transcription factors.

Mesoblast's allogeneic mesenchymal precursor cell (MPC) therapy is entering Phase II trials in early 2009 to repair nonunion of long bone fractures.

The safety of the viral vectors used in the Spiegelman and Melton studies is a major concern, said Kai Pinkernell, head of research of Cytori Therapeutics Inc. He also said therapies that irreversibly alter cellular identity could be a hard sell for regulators.

"If it's not reversible, what are you going to do if there's a bad safety outcome?" asked Pinkernell.

Cytori is developing devices to process and purify adult stem and regenerative cells from adipose tissue. Cytori's Celution System adipose graft device is in Phase II trials for reconstructive surgery following partial mastectomy.

\section{REFERENCES}

1. Seale, P. et al. Nature; published online Aug. 20, 2008; doi:10.1038/nature07182

Contact: Bruce Spiegelman, Dana-Farber Cancer Institute, Boston, Mass. e-mail: bruce_spiegelman@dfci.harvard.edu

2. Tseng, Y.H. et al. Nature; published online Aug. 20, 2008; doi:10.1038/nature07221

Contact: Yu-Hua Tseng, Harvard Medical School, Boston, Mass. e-mail: Yu-Hua.Tseng@joslin.harvard.edu

3. Zhou, Q. et al. Nature; published online Aug. 27, 2008; doi:10.1038/nature07314

Contact: Douglas Melton, Harvard University, Cambridge, Mass. e-mail: dmelton@mcb.harvard.edu

4. Seale, P. et al. Cell Metab. 6, 38-54 (2007)

5. Slack, J.M. Nat. Rev. Mol. Cell Biol. 8, 369-378 (2007)

6. Horb, M.E. et al. Curr. Biol. 13, 105-115 (2003)

COMPANIES AND INSTITUTIONS MENTIONED

Acceleron Pharma Inc., Cambridge, Mass. Cytori Therapeutics Inc. (NASDAQ:CYTX; Xetra:XMPA), San Diego, Calif.

Dana-Farber Cancer Institute, Boston, Mass. GlaxoSmithKline plc (LSE:GSK; NYSE:GSK), London, U.K. Harvard Medical School, Boston, Mass. Joslin Diabetes Center, Boston, Mass.

Mesoblast Ltd. (ASX:MSB), Melbourne, Australia

Novocell Inc., San Diego, Calif.

Sangamo BioSciences Inc. (NASDAQ:SGMO), Richmond, Calif. Stryker Corp. (NYSE:SYK), Kalamazoo, Mich.

Takeda Pharmaceutical Co. Ltd. (Tokyo:4502), Osaka, Japan

University of Minnesota, Minneapolis, Minn.

University of Pennsylvania, Philadelphia, $\mathrm{Pa}$. 